\title{
LOS CORREGIDORES DE ALICANTE. PERFIL SOCIOLÓGICO Y POLÍTICO DE UNA ÉLITE MILITAR
}

\author{
Por Enrique GIMÉNEZ LÓPEZ
}

Universidad de Alicante

En el nuevo orden político instituido en Valencia tras el decreto de 29 de junio de 1707 por el que se abolía el régimen foral, la institución corregimental ocupaba un lugar relevante. Desarrollado en Castilla como agente del absolutismo (1), transvasado a América para afirmar la autoridad colonial, la concepción uniformista que impregnó el centralismo borbónico hizo del corregidor el instrumento idóneo para aplicar en los territorios de la antigua Corona aragonesa la legislación castellana y someter a los reinos declarados rebeldes al nuevo orden nacido de la victoria de 1714 .

El carácter marcadamente intimidatorio que tuvo el cargo en Aragón, Cataluña y Valencia le otorgó un fuerte sesgo militarista, que en Valencia y Cataluña supuso un predominio casi absoluto de altos oficiales del ejército en el desempeño de los corregimientos ${ }^{(2)}$, y un poder relevante al Capitán General, situado en el vértice de la nueva estructura institucional, tras unos balbuceos iniciales ${ }^{(3)}$. La mayor parte de los corregidores valencianos y catalanes, y un número importante de los aragoneses eran, al mismo tiempo, gobernadores militares. Por tanto, representaban al rey y al Consejo de Castilla, actuaban como delegados del Capitán General respectivo en cuestiones relativas a la milicia y, complementariamente, representaban al Intendente General como Subdelegados de Rentas. En Valencia y Cataluña, las propuestas de candidatos a cubrir vacantes se realizaban por la Secretaría de Guerra, y sólo tras el decreto que designaba nuevo gobernadorcorregidor intervenía la Cámara de Castilla, reduciéndose su cometido a conceder el preceptivo despacho de corregidor que facultaba el desempeño de las funciones puramente judiciales y de administración civil: presidir la corporación municipal de la capital del corregimiento y sus Juntas de Propios y Arbitrios, o cualesquiera otras, y promulgar ordenaciones municipales y sentencias en primera instancia. 
Las muy variadas tareas encomendadas al corregidor militar, desde las puramente represivas a las de fomento económico, confieren un interés muy particular a quienes llevaron a cabo estas funciones como agentes del poder real. Trazar su perfil sociológico y la relevancia del cargo en su cursum honorum no es, sin embargo, tarea fácil por la dispersión de las fuentes y por las propias noticias que éstas ofrecen, no siempre idóneas respecto al objetivo propuesto. Las hojas de servicios de oficiales y suboficiales, conservadas en el Archivo General de Simancas, que tan interesantes resultados proporcionaron a las investigaciones de Francisco Andújar para el siglo XVIII ${ }^{(4)}$, y a Fernández Bastarreche para el siglo XIX ${ }^{(5)}$, nos han sido de escasa utilidad en nuestro trabajo sobre los corregidores en el siglo XVIII valenciano, por hacer referencia las hojas localizadas a servicios desempeñados en momento iniciales de la carrera militar de los corregidores, cuando no habían sobrepasado el grado de suboficial.

De gran utilidad para reconstruir cronológicamente la lista de los corregidores que actuaron en Alicante desde el fin de la Guerra de Sucesión hasta $1833^{(6)}$, son los documentos conservados en la sección de Consejos Suprimidos del Archivo Histórico Nacional, en la serie correspondiente a Gracia y Justicia de Aragón. Proporcionan esencialmente noticias sobre la fecha de designación, toma de posesión y de traslado. Uno de ellos, el signado con el número 18.541, fue utilizado por el profesor Eduardo Escartín para su comunicación «El corregiment de Barcelona: notes per al seu estudi» (7).

Pero es en los fondos de la Secretaría de Guerra, en Simancas, donde se encuentra la documentación más interesante, por cuanto ofrece noticias sobre el desempeño del cargo, aun cuando la localización es muy trabajosa al hallarse dispersa, y ello ha sido la causa, quizá, de que no se haya utilizado para el estudio de la administración corregimental borbónica en los reinos de la antigua Corona aragonesa.

\section{LOS GOBERNADORES-CORREGIDORES}

La derrota de Almansa en los últimos días de abril de 1707 y la capitulación de la capital valenciana durante el mes siguiente, supuso el control militar del antiguo Reino por el ejército de Berwick. De forma sucesiva cayeron en manos borbónicas Alcira, Játiva y otras plazas. Alicante, bien defendida por su fortaleza y por tropas inglesas al mando del Mayor John Richards, se preparó para un largo asedio.

Mientras Berwick aprovechaba el empuje logrado en Almansa y llegaba a orillas del Ebro, el Caballero D'Asfeld quedaba en territorio valenciano con parte del ejército borbónico para acabar con los focos de resistencia que habían quedado en su retaguardia, algunos de indudable importancia militar por su condición de plazas fuertes, como Denia o Alicante. 
El primer asedio de Denia, defendida por Diego Rejón, marqués de Alcantarilla, fracasó, y Alcoy fue tomada en los primeros días de $1708^{(8)}$. Denia pasó definitivamente a manos de D'Asfeld en noviembre de $1708^{(9)}$, quedando Alicante como la única plaza valenciana de entidad en poder aliado.

El 30 de noviembre, las tropas del caballero D'Asfeld llegaron frente a la ciudad, defendida por 800 miqueletes y 400 soldados españoles. En el castillo se hallaban 800 ingleses del regimiento de infantería de Hotham, el regimiento Sybourg y un destacamento de artilleros y marinos.

La ciudad capituló pronto, permitiéndose a la guarnición española embarcarse para Cataluña, quedando sólo los ingleses en la fortaleza ${ }^{(10)}$, a la espera de que la escuadra del almirante Byrg los socorriera. Para lograr la rendición del castillo fue utilizada la más avanzada técnica en ingeniería militar: la construcción de una mina, que estalló en la cara oeste de la fortaleza el 3 de marzo de 1709. El 19 de abril las tropas borbónicas recuperaban para el rey Felipe la última plaza valenciana, y el 13 de mayo el monarca comunicaba al gobernador del Consejo de Castilla el nombramiento como primer corregidor de Alicante del Coronel Patricio Miset, quien había sido designado gobernador militar con anterioridad a propuesta del propio D'Asfeld. Al igual que se había efectuado con el resto de poblaciones valencianas, la Cámara de Castilla debía entregar el necesario despacho a Miset para que éste actuara como corregidor ${ }^{(11)}$.

Sólo unos pocos meses permaneció Miset en Alicante. La toma de posesión del corregimiento la efectuó ante el oidor de la Chancillería de Valencia Tomás Melgarejo el 26 de junio ${ }^{(12)}$, pero a fines de ese mismo año Miset fue ascendido a Brigadier y trasladado al ejército que operaba en Extremadura ${ }^{(13)}$.

$\mathrm{Al}$ igual que en el resto de los corregimientos valencianos el desconcierto y la desorganización más completas dominaban la situación postbélica en Alicante. La indefinición administrativa en muchos aspectos del gobierno valenciano era, todavía en 1709 , considerable. A fines de 1708 , el rey había rechazado la planta corregimental diseñada por el presidente de la Chancillería valenciana, Pedro de Larreategui ${ }^{(14)}$, ordenando que los gobernadores militares recibieran de la Cámara los títulos de corregidores, pero en todos los ambientes se estimaba que mientras prosiguiera la guerra estas decisiones eran coyunturales. La posibilidad de que las gobernaciones forales sirvieran de base para la nueva ordenación política del territorio valenciano, condujo a Alicante a solicitar la capitalidad de la antigua gobernación de Orihuela, en lugar de su vieja rival del sur, petición apoyada por el corregidor Miset:

«El Gobernador y Corregidor que es y fuere de la dicha Ciudad se intitula gobernador de Alicante y Reino de Valencia, de Jijona a esta parte, como se intitulaba antes el que lo era de Orihuela, y que el dicho Gobernador o Corregidor tenga toda la jurisdicción desde la dicha ciudad de Alicante en toda la referida Gobernación, incluyéndose también en ella la dicha Ciudad de Orihuela y todos los lugares que eran de su distrito, y que a más del gobierno de la dicha Ciu- 
dad de Alicante se añada el de Jijona, villas de Castalla, Onil, Ibi, Tibi y Villajoyosa» ${ }^{(15)}$.

Los afanes expansionistas de Alicante y su primer corregidor eran incompatibles con la nueva distribución corregimental, en la que criterios políticos primaban sobre la funcionalidad administrativa. Orihuela, sede episcopal y antigua capital de la gobernación foral, contaba con un gobernador militar con despacho de corregidor; Jijona, por su fidelidad a Felipe $\mathrm{V}$ habia recibido el título de ciudad y el privilegio de encabezar una gobernación que abarcaba las poblaciones de la Hoya de Castalla ${ }^{(16)}$. El deseo de incorporar Jijona y el territorio de su gobernación al corregimiento de Alicante, expresado por Miset, se mantuvo a lo largo del siglo XVIII como un proyecto deseable y defendido por quienes pretendieron racionalizar el mapa corregimental valenciano, como se hizo constar en el proyecto de reforma elaborado por la Audiencia en $1721^{(17)}$, o en el realizado por el Capitán General Duque de Caylus en $1753^{(18)}$.

Patricio Miset fue el único corregidor de Alicante que alcanzó el cargo con el grado de Coronel, si bien cuando pasó a Extremadura lo hizo como Brigadier. Los restantes lo hicieron con mayor graduación: siete como Brigadier, de los que cuatro lo fueron entre 1814 y 1833; diez como Mariscales de Campo, y cuatro como Tenientes Generales. Algunos consiguieron su ascenso a Teniente General mientras servían el corregimiento de Alicante: el Marqués de Alós, corregidor entre 1746 y 1759; José Ladrón de Guevara, que ocupó el corregimiento entre 1760 y 1767, y Francisco Javier Pacheco, que lo hizo entre 1786 y 1793. Alejandro de la Motte fue corregidor entre 1727 y 1734 como Mariscal de Campo; pasó al gobierno de Málaga, y en 1741 fue designado nuevamente corregidor de Alicante, si bien ahora con el grado de Teniente General ${ }^{(19)}$.

Con frecuencia el gobernador de Alicante era el oficial de más alta graduación en el Reino tras el Capitán General. Esta circunstancia llevó a que, en algunos casos de fallecimiento del Capitán General, fuera el gobernador militar de Alicante quien asumiera interinamente la Capitanía. Así, Gerónimo Conde, secretario del Capitán General Manuel de Sada, informó a Esquilache de la gravedad de la primera autoridad valenciana, comunicándole que había informado de la situación al gobernador de Alicante, el Teniente General José Ladrón de Guevara, «respecto a ser el oficial de mayor graduación» (20). Cuando Sada falleció el 2 de febrero de 1764, Ladrón de Guevara pasó a desempeñar el mando militar de Valencia y Murcia hasta la toma de posesión del Conde de Aranda (21).

Un procedimiento semejante se siguió durante la enfermedad y muerte del Capitán General Juan Francisco Martí, marqués de Vanmarck. Informado de su gravedad, el Teniente General Jorge Dunant solicitó el mando accidental por ser el el Teniente General más antiguo ${ }^{(22)}$, y en efecto desempeñó el cargo durante el período comprendido entre la muerte de Vanmarck y la toma de posesión del marqués de Croix. En el interín se cursaron órdenes a todos los gobernadores militares para que enviaran toda la correspondencia referente a asuntos del servicio a Dunant, y en Valencia, el Teniente de Rey de la capital y el secretario de la Ca- 
pitanía quedaron encargados de abrir el correo para su distribución, pasando al Regente de la Audiencia los papeles correspondientes a la administración civil y remitiendo a Dunant los relativos a los asuntos castrenses ${ }^{(23)}$.

Todos ellos eran nobles, si bien algunos pertenecian a la aristocracia, como el conde de Roydeville, el marqués de Villareal, el Marqués de Alós padre e hijo, y el Conde de Baillencourt, y la mayoría eran caballeros de alguna de las órdenes militares, lo que no era óbice para que solicitaran encomiendas cuando se producía una vacante: José de Chaves solicitó una encomienda de la orden de Santiago que había quedado vacante por fallecimiento del Teniente General de la Croix ${ }^{(24)}$, también solicitada por Alejandro de la Motte ${ }^{(25)}$.

Debido a la composición plurinacional de los ejércitos, era usual que se apreciara la lealtad al soberano por encima del origen nacional, por lo que es frecuente encontrar un alto nivel de internacionalidad en la alta oficialidad borbónica ${ }^{(26)}$. En el caso de los corregidores alicantinos encontramos militares flamencos, como el Conde de Roydeville, cuyo patrimonio había sido confiscado en Flandes ${ }^{(27)}$, o Alejandro de la Motte, quien había iniciado sus servicios en los Países Bajos españoles e intervenido en la batalla de Echeren, cerca de Amberes, pasando a España con el regimiento de Guardias Walonas ${ }^{(28)}$; franceses, como el Conde de Baillencourt o el Brigadier Fernando de Saint-Croix; y suizos, como el Teniente General Jorge Dunant, originario de Saint-Gall, y que llegó a España en 1710 como mercenario al servicio de Luis XIV para pasar, más tarde, a servir a los borbones españoles.

Casi todos ellos tenían una dilatada experiencia bélica, habiendo intervenido en las campañas más destacadas de su tiempo y, en algunos casos, su mandato se veia temporalmente interrumpido, o su toma de posesión postpuesta, como consecuencia del encargo de una misión militar. El Conde de Roydeville, nombrado corregidor de Morellá en junio de 1718 no tomó posesión hasta septiembre de 1721 por hallarse en las expediciones de Sicilia y Africa ${ }^{(29)}$; y el 10 de marzo de 1742, el Teniente General Alejandro de la Motte recibió orden de abandonar temporalmente el corregimiento de Alicante para encargarse del mando de Orán ${ }^{(30)}$.

La Guerra de Sucesión fue durante la primera mitad de siglo referencia obligada en la relación de méritos de los corregidores militares: José de Chaves, corregidor entre 1715 y 1722 , había sido hecho prisionero por los austracistas y encarcelado en la ciudad de la que sería gobernador años más tarde ${ }^{(31)}$; Antonio Alós, marqués de Alós, inició su carrera militar en la Guerra de Sucesión, llegando a ser ayudante del duque de Berwick ${ }^{(32)}$; Jorge Dunant, designado en 1775 corregidor de Alicante a los 81 años de edad, intervino en el sitio de Gerona a primeros de 1711 y en el asalto de Barcelona en $1714^{(33)}$.

Los restantes hechos de armas en los que participó España en Italia, norte de Africa, Portugal o los sitios de Gibraltar durante el siglo XVIII, aparecen destacadamente en las relaciones de servicios de estos corregidores: Alejandro de la Motte participó, con su regimiento de Guardias Walonas, en las expediciones a Cerdeña y Ceuta, y en 1719 se encontraba defendiendo San Sebastián frente a 
los franceses; Antonio de Alós logró rápidos ascensos en las campañas de Italia y el marquesado de Alós por su contribución a la conquista del reino de las Dos Sicilias; su hijo, José de Alós, también corregidor de Alicante entre 1797 y 1798, había participado en la recuperación de Menorca y en el sitio de Orán ${ }^{(34)}$; la extensa vida militar del Teniente General Jorge Dunant no quedaba ajena a casi la totalidad de los acontecimientos bélicos de la primera mitad de siglo, como el sitio de Gibraltar en 1726, la expedición a Orán de 1732, la campaña de Italia, para la que formó su propio regimiento en Saboya en 1743, y la campaña de Portugal de 1762, donde llegó a desempeñar el cargo de gobernador militar de Chaves ${ }^{(35)}$.

El caso del Teniente General Antonio Oliver, corregidor entre 1783 y 1786, es excepcional. Se trata de un militar cuya carrera se halla vinculada a tareas administrativas, con limitadas experiencias en campaña. Iniciada su carrera militar como cadete en 1734, intervino en Italia con su regimiento de Infantería de Soria, para pasar en 1740 al regimiento de Infantería de León, con destino en Ceuta. En 1745 ingresó en la administración militar, desempeñando desde ese año la Secretaría de la Comandancia General de Cataluña. Dos años más tarde, el marqués de la Ensenada lo agregó a la Secretaría del Despacho de la Guerra, de la que era titular, ascendiendo a Teniente Coronel en 1755 y a oficial segundo de dicha Secretaría. En 1760, Ricardo Wall le nombró secretario de la Junta encargada de redactar unas nuevas ordenanzas militares, ascendiendo ese mismo año a Coronel. Abandonó la administración central en 1770 con el grado de Brigadier para desempeñar la Capitanía General de Yucatán. A su vuelta, y ya Mariscal de Campo, tuvo su primera experiencia bélica desde sus días de Cadete, interviniendo en el bloqueo de Gibraltar ${ }^{(36)}$. Ascendido a Teniente General en 1783, fue designado en abril de ese año corregidor de Alicante con la recomendación del Secretario de Guerra y Hacienda, el navarro Miguel Muzquiz ${ }^{(37)}$, para ser promocionado en 1786 a cargos de mayor relieve.

La gobernación de Alicante, la plaza militar valenciana de mayor valor estratégico, suponía haber alcanzado uno de los más altos escalones de la carrera militar. Cuando Antonio de Alós agradeció desde Nápoles al marqués de la Ensenada su designación como corregidor de Alicante, lo consideró como premio a sus treinta y siete años de servicios, manifestando su satisfacción a su favorecedor en los siguientes términos:

«Sólo Vuestra Excelencia sabe dar más de lo que se pide; me dicen todos que es mejor destino el de Alicante que Tortosa» ${ }^{(38)}$.

Algunos corregidores llegaron a alcanzar, tras su paso por Alicante, distintas capitanías generales: José de Chaves pasó a desempeñar la balear en 1721; Antonio de Alós, tras ocupar la gobernación de Gerona, fue designado Capitán General de Baleares en 1767; y Francisco Javier Pacheco, ya como Teniente General, dejó Alicante el 13 de febrero de 1793 para ocupar interinamente la Capitanía General de Galicia ${ }^{(39)}$. Otros pasaron a desempeñar gobiernos para los que se requería una experiencia contrastada: el Brigadier Fernando Pinacho ocupó 
el corregimiento de Tortosa en 1715 como uno de los primeros corregidores nombrados para Cataluña después de $1714^{(40)}$, y el Conde de Roydeville fue trasladado desde Alicante a un corregimiento de la dimensión militar y política del de Barcelona ${ }^{(41)}$.

Pero en la mayoría de los casos, el gobierno de Alicante suponía el colofón a toda una larga carrera militar. Así sucede con José Ladrón de Guevara, corregidor entre 1760 y su fallecimiento en 1767; con el Conde de Baillencourt, muerto en 1775 tras ocho años de servir el corregimiento; con Jorge Dunant, que ocupó el gobierno de Alicante entre 1775 y 1783; y José Antonio Romeo, fallecido en 1797 tras cuatro años de corregidor.

El hábito muy extendido de considerar los corregimientos militares como puestos vitalicios y, por tanto, no sometidos a la limitación trienal de los corregimientos civiles, tenía consecuencias políticas negativas, entre ellas el deterioro físico lamentable que la avanzada edad causaba entre los corregidores. En 1766, un «insulto aplopéjico» dejó paralizado el lado derecho del corregidor José Ladrón de Guevara, con efectos políticos graves que más adelante comentaremos; el conde de Baillencourt, desde su toma de posesión en agosto de 1767 , se hallaba delicado de salud, necesitando pasar a Aguas de Busot para tomar baños, por lo que los asuntos militares eran resueltos habitualmente por el Teniente de Rey de Alicante ${ }^{(42)}$, y los administrativos y judiciales por su Alcalde Mayor. Fue José Fernández de Valdivia, segundo jefe militar de Alicante, quien informó en septiembre de 1775 de la situación de invalidez en que se encontraba el corregidor, «incapaz de articular palabra», al Secretario de Guerra, conde de Ricla ${ }^{(43)}$, estado en el que se mantuvo hasta su muerte a fines de noviembre de ese mismo año ${ }^{(44)}$. Jorge Dunant, que tomó posesión del corregimiento contando 83 años de edad ${ }^{(45)}$, había solicitado Alicante por "haberme probado siempre bien el clima» (46); cuando solicitó el retiro a los 87 años aduciendo sus muchos achaques y avanzada edad, le fue denegado «por no querer el Rey tener otro gobernador en aquella plaza en tiempo de guerra» ${ }^{(47)}$, falleciendo finalmente en 1783 sin que se hubieran atendido sus deseos de cese.

El estado de ceguera, sordera y senilidad del Teniente General confirió a su secretario, el escribano José Gentil, un poder considerable. Denunciado en un escrito apócrifo de haberse enriquecido abusando de la confianza del corregidor, dedicándose al contrabando y a juegos de banca, fue sometido a investigación. El secretario se defendió manifestando que eran calumnias de quienes se consideraban sus enemigos «por ser persona de la confianza del Gobernador». El Alcalde Mayor, sin embargo, recomendó su separación del cargo "por lo extendido que están en Alicante los juegos prohibidos», lo que no prosperó por la insistencia de Dunant a que su secretario continuara a su servicio, "por ser sujeto de su confianza y que sin él no puede desempeñar sus encargos por la confianza, desempeño y fidelidad con que lo hace») ${ }^{(48)}$.

Lo habitual de las enfermedades, ausencias, traslados y fallecimientos hacía que las interinidades alcanzaran una buena importancia política. El primer Alcal- 
de Mayor que tuvo la ciudad, nombrado en 1709 por Patricio Miset, el abogado Francisco Esteban Zamora y Cánovas, alcanzó en gran parte del primer reinado de Felipe $V$ un gran poder en la ciudad, resultado de sus frecuentes actuaciones como corregidor interino. Entre 1720 y 1722 fue sometido a una extensa investigación para responder a acusaciones muy graves de abuso de poder y prevaricación, de las que salió absuelto ${ }^{(49)}$.

En ocasiones, la ausencia de corregidor coincidia con la falta de Alcalde Mayor, con lo que el regidor decano pasaba a desempeñar la jurisdicción ordinaria, lo que no era del agrado de la Cámara de Castilla: en agosto de 1726 marchó con licencia a Santander el corregidor Conde de Roydeville, con el encargo de comandar las tropas de Cantabria; al no haber sustituto del controvertido Alcalde Mayor Francisco Esteban Zamora, la jurisdicción recayó en el regidor decano Pedro Burgunyo. La aprobación real instaba a que el corregidor propusiera Alcalde Mayor y, en caso contrario, el Consejo tomaría las oportunas providencias ${ }^{(50)}$.

La designación de Alejandro de la Motte como gobernador de Orán en marzo de 1742, no supuso el abandono del cargo de corregidor de Alicante, por lo que fue sustituido interinamente. El Capitán General de Valencia, Duque de Caylus, manifestó a Campillo su deseo de que se nombrara a un militar para corregidor interino, ya que se evitaba una previsiblemente dilatada situación transitoria con un Alcalde Mayor como responsable de los asuntos civiles, y un Teniente de Rey al mando de los aspectos castrenses de la plaza:

«Y suponiendo que su ausencia será larga convendría mucho que al Teniente de Rey Juan Díez de Real se le encargase el Corregimiento de la ciudad de Alicante interinamente por ser oficial de toda satisfacción, vigilante y celoso del servicio, con cuya providencia no quedaría aquel gobierno político al cuidado de un Alcalde Mayor nuevo" (5).

La desconfianza mutua entre civiles y militares en el mando corregimental, explica el interés del Capitán General de Valencia por cubrir las interinidades con militares, posibilitando que el poder civil y castrense se mantuvieran unidos en manos de la alta oficialidad. Entre 1742 y 1746, el corregidor de Alicante fue el Mariscal de Campo Marqués de Villareal, si bien como interino ${ }^{(52)}$, y a su sustituto, el Marqués de Alós, le fue confiado el corregimiento en interín, «con la propiedad del referido empleo para cuando vaque» ${ }^{(53)}$.

Idéntico argumento al del Duque de Caylus respecto a la solvencia en mantener unida la jurisdicción civil y la militar, era utilizado por el Teniente de Rey de Alicante tras la muerte del corregidor José Ladrón de Guevara. Tras ocho meses de desempeñar el mando militar de la plaza, a la espera de la llegada del nuevo gobernador, Conde de Baillencourt, el Coronel José Gutiérrez de Valdivia solicitaba que en los casos de vacante los Tenientes de Rey, o segundos comandantes de las plazas, ejercieran también el mando civil, «ejerciendo en todos los actos las funciones de tal Corregidor, con la presidencia del Ayuntamiento, evitando 
por este medio las disputas que me dicen, estando divididas las jurisdicciones, de lo que resulta un perjudicial atraso en el servicio de V. Mag.» ${ }^{(54)}$.

Sin embargo, los corregidores alicantinos eran ante todo gobernadores militares de una plaza fuerte, y sus obligaciones civiles estaban en un segundo y, en ocasiones, molesto plano. El ejemplo más ilustrativo es el del Mariscal de Campo Juan de Cereceda, a quien Felipe $\mathrm{V}$ designó Corregidor de Alicante interín ocupara la Capitanía General de Baleares el corregidor titular José de Chaves ${ }^{(55)}$. Cereceda obedeció disciplinadamente, llegando a Alicante el 28 de abril de 1728, y unos pocos días después escribió cartas al Marqués de Castelar y al Marqués de Grimaldo solicitando su sustitución «por ser la actividad de mandar paisanos lo que más aborrezco» ${ }^{(56)}$, y que mientras no tuviera oportunidad de actuar en campaña deseaba permanecer en su casa sin cargos políticos, para los que que se consideraba falto de las dotes necesarias:

«Aseguro a V. E. no es para mi genio cosa de gobierno en parte alguna y que me considero incapaz de mandar otra cosa que soldados en campaña, y después de hacer el debido aprecio de tan singular honra, teniendo por muy cierto que dicho don José de Chaves no volverá a este Gobierno, no excuso suplicar a V. E. (...) se sirva mandar venga aquí persona que gobierne esto, que cualquiera lo hará mejor que yo») ${ }^{(57)}$.

Cuando a finales de mayo Baltasar Patiño, Marqués de Castelar, le requirió por escrito si deseaba mantenerse o no en el mando interino de Alicante, la respuesta de Cereceda fue tan clara como lo habían sido sus anteriores solicitudes de renuncia: «más bien apetezco mandar cuatro soldados con desconveniencia que millares de paisanos en el mejor gobierno» ${ }^{(58)}$.

La tardanza en obtener en la Cámara de Castilla el despacho que les facultaba para actuar como corregidores, denota el escaso aprecio que sentían por la dimensión civilista de función y provocó las protestas de la Cámara en los años inmediatamente posteriores a la Nueva Planta, deseosa de ejercer algún control, aunque fuera mínimo, sobre los corregidores valencianos. En marzo de 1710, la Cámara se quejaba de que el Brigadier Fernando Pinacho hubiera comenzado a ejercer como Corregidor sin haber obtenido el preceptivo despacho ${ }^{(59)}$, y en 1715 multó al Mariscal de Campo José de Chaves con 60 ducados por su morosidad en tomar posesión.

La resistencia de los altos oficiales a jurar su cargo ante el presidente de la Chancillería, como lo exigía la Cámara, y no ante el Capitán General como era su deseo, fue también fuente de problemas hasta que, reducida la Chancillería a Audiencia en 1716, el Capitán General pasó a presidir el Real Acuerdo. El mismo José de Chaves juró su cargo ante el Marqués de Villadarias, Capitán General, pese a que la Cámara de Castilla pretendía que lo efectuase ante Juan de Valcárcel, último presidente de la Chancillería valenciana, al considerar que «el Mariscal Chaves tiene un cargo político y de justicia» ${ }^{(60)}$. 


\section{LA ACTUACIÓN CORREGIMENTAL}

La superior relevancia de la dimensión militar del cargo, ejercido por oficiales de alta graduación con escasa experiencia en el trato con civiles, con hábitos de mando adquiridos en acuartelamientos o en campaña, daba lugar a frecuentes abusos de autoridad, conflictos con las autoridades locales y, en algunos casos, a actuaciones teñidas de brutalidad. A diferencia de los corregidores civiles, sujetos al denominado juicio de residencia, y limitado temporalmente su mandato a un trienio, los militares se resistieron eficazmente a someterse a prestaciones de responsabilidad civil, detentando el cargo sin limitación temporal alguna, razones por las que su poder efectivo era muy considerable, viéndose reforzado notablemente su función de instrumento de autoridad que conllevaba el cargo.

La utilización de esa autoridad con fines privados en Alicante queda patente en el conflicto que enfrentó a la poderosa familia de los Canicia con el Teniente General Marqués de Alós durante 1758. Los Canicia formaban una de las familias más influyentes y poderosas de la ciudad. Antonio Rotlá y Canicia fue designado regidor noble en el primer ayuntamiento borbónico de Alicante ${ }^{(61)}$, y su hijo Luis Rotlá y Canicia y nieto Antonio Canicia también fueron regidores, hallándose emparentados con los Marqueses del Bosch y con los Condes de Torrellano ${ }^{(62)}$.

Los problemas surgieron por diferencias de trato, al negar algunas mujeres de la familia Canicia el tratamiento de Excelencia al Marqués de Alós y esposa cuando éste fue ascendido a Teniente General. No era una cuestión baladí, considerada en el contexto social en que se producía y dada la relevancia política de quienes entraban en conflicto: un Corregidor dotado de alto rango oficial, y una familia con una tradicional posición de poder en la ciudad, en la que venía desempeñando funciones de gobierno municipal desde el siglo XVI ${ }^{(63)}$. La afabilidad en los saludos era la confirmación del prestigio y su posición de poder de quien lo recibía ${ }^{(64)}$, por lo que una serie de incidencias en el trato entre el Corregidor y miembros de la familia Canicia derivó en problema político.

Los hechos que conocemos giran en torno a cuestiones de conducta: en una ocasión el corregidor encarcela a los cocheros de los Canicia porque al pasar junto al que conducía a la corregidora «dijo que era un desacato por haberle echado el polvo encima»; en otra, levanta su bastón contra otro cochero de los Canicia amenazándole con golpearle si al verlo en la calle no paraba su carruaje; o increpa a doña Mariana Vaillo de Llanos, esposa de Antonio Canicia e hija del Conde de Torrellano, llamándole «desatenta y mal criada» por no saludarle debidamente al verle en la colegiata ${ }^{(65)}$.

En enero de 1758, el Marqués de Alós ordenó la detención del regidor Antonio Canicia y de su hermano Luis, acusándolos de haber intervenido en una pelea callejera y haber propinado golpes a un criado. Ante la intercesión del Conde de Villafranqueza para evitar su ingreso en prisión, el Corregidor puso como condición que se lo pidiesen Mariana Vaillo, mujer de Antonio Canicia, y Lorenza Pas- 
cual de Ibarra, madre de los detenidos, y que le dieran el tratamiento de Excelencia, lo que suponía un reconocimiento de su prestigio y de su preeminente posición de poder. La respuesta negativa de ambas mujeres provocó la ira del corregidor, que no veía confirmado su rango, enviando a los dos hermanos a los castillos de Santa Pola y Guardamar, y considerando el empecinamiento de ambas mujeres «efectos todos de vanidad, locura y mala crianza, que exceden al cariño de madre y mujer» ${ }^{(66)}$.

La familia Canicia acudió a la Real Audiencia de Valencia y denunció los hechos ante el Capitán General y el Rey, a quienes advertía el mal ejemplo que los abusos de Alós provocaba:

«... por sus fines particulares está dando alientos a los plebeyos para que se atrevan y desprecien a los nobles, y nobles que han sabido ser el lustre y distinción de la patria.... ${ }^{(67)}$.

En torno al incidente se constituyó un frente de oposición al corregidor del que formaba parte el propio obispo de Orihuela, Juan Elías Gómez de Terán, quien había tenido frecuentes roces con las autoridades políticas, lo que fue aducido por el Corregidor en su descargo:

«Este prelado, enseña la experiencia, se opone a toda autoridad real, como es notorio en tan repetidos lances, y sólo quieren que residan en él todos los respetos y temores del pueblo» ${ }^{(68)}$.

A fines de marzo, una orden real prohibía al Marqués de Alós hablar sobre su contencioso con los Canicia, en lo que el Corregidor entendió como un éxito de los que se oponían a su gestión: el obispo aprovechaba el momento para debilitar la autoridad real a través de sus ataques contra el representante del monarca en Alicante, añadiendo el Marqués de Alós que «el genio de este prelado es tan violento como notorios sus hechos, y buscando la casa Canicia su apoyo ha disparado contra mí»; el Teniente de Rey también se valía de la situación, en opinión del Corregidor, para menoscabar la autoridad del gobernador, señalándolo Alós como un activo elemento de lo que él consideraba una conspiración contra sus competencias políticas ${ }^{(69)}$.

El dictamen del magistrado Gil de Jaz fue muy crítico hacia las actuaciones del Corregidor, quien fue calificado de imprudente al proponerse intimidar a los Canicias y por su decisión de no dar cumplimiento a los despachos expedidos por la Audiencia ordenándole poner en libertad a los detenidos. En sus consideraciones el magistrado lo consideraba culpable «porque su carácter de Teniente General lo debió haber retraído de empeñarse tan ruidosamente en una pretensión tan poco sólida como la del tratamiento, y especialmente con mujeres de una distinguida familia» ${ }^{(70)}$, y nunca debió utilizar sus prerrogativas para cuestiones de índole personal, ni cometer el abuso de encarcelar a dos caballeros no militares ignorando todo procedimiento judicial.

La propuesta del magistrado, confirmada posteriormente por el monarca, abogaba por la restitución a su casa de los hermanos Canicia; una amonestación 
al Corregidor, previniéndole que su conducta había sido del desagrado del rey $\mathrm{y}$, finalmente, la condición más humillante para Alós:

«... que restituidos los arrestados en su casa los visite el Marqués

en ella, como por especie de desagravio, sin expresión que lo ejecuta mandado, ni hablar de lances pasados» ${ }^{(71)}$.

La inclusión de este castigo de etiqueta, sin duda el de más duro cumplimiento, recalcaba la importancia de las relaciones formales en la conducta política, y cómo un error de dosificación en el uso del poder encomendado al Corregidor podía depararle desagradables consecuencias si su enfrentamiento se producia con personas que también detentaban parcelas de poder en otros ámbitos. Antonio Alós, meses más tarde, cesaría en el corregimiento de Alicante al ser designado Gobernador político y militar de Gerona y Comandante General del Ampurdán ${ }^{\text {(72) }}$.

Otro caso de actuación abusiva fue el encarcelamiento de los regidores nobles Juan Pascual de Pobil y Esteban Rovira en 1766 por el Corregidor José Ladrón de Guevara. En mayo de aquel año, el Teniente General Ladrón de Guevara, con cincuenta y tres años de servicios en el ejército, sufría un ataque que le dejó paralizado el brazo derecho, por lo que solicitó permiso para el uso de estampilla al estar imposibilitado para la firma. La corporación municipal, reunida en Cabildo, acordó en las sesiones del 30 de mayo y 2 de junio solicitar la sustitución del Corregidor por el Alcalde Mayor ${ }^{(73)}$, enviando un escrito a Manuel de Roda en los siguientes términos:

«En atención a los graves perjuicios que seguirían a esta Ciudad y Común de semejante facultad (uso de la estampilla) por el manejo que de ello pudieran hacer otros por el estado en que dicho Excmo. Sr. Corregidor se halla de no poder asistir al despacho de los negocios ni entender en ellos por sus continuos y repetidos transtornos» ${ }^{(74)}$.

La reacción del Corregidor, que por entonces actuaba como Capitán General Interino de Valencia, fue violentísima. La solicitud de destitución fue titulada «escandalosa injuria hecha a un oficial general», y que el Ayuntamiento pretendía desembarazarse de la autoridad militar para ejercer su control ilimitado sobre la ciudad:

«El espíritu que fomenta y proyecta tan ruidosas cavilaciones no es otro que el de una desenfrenada propensión a vivir con libertad, sin reconocer superior que con entereza contenga las vivezas que les dicta la poca cordura apoyada de la más refinada envidia de este Alcalde Mayor por su notoria ambición y querer abarcar lo de Guerra, Rentas, Correos, Pósitos y demás ramos que estén a mi cargo» ${ }^{(75)}$.

En el mismo escrito dirigido a Roda, el Corregidor informaba que «para evitar mayores inconvenientes» había ordenado el arresto en el castillo de Santa Bárbara de la misma ciudad, de los regidores Juan Pascual de Pobil y Esteban Rovira, primos hermanos, dos de los «principales motores» de la conspiración contra él.

La razón de fondo del conflicto entre regidores y Corregidor se hallaba en 
el proceso de las primeras elecciones para Síndico Personero y Diputados del Común, ya que unos y otros deseaban lograr candidatos afines para esos puestos. El Corregidor decía tener datos de que algunos regidores repartían papeletas ya escritas con los nombres que debían elegirse, figurando en ellas personas vinculadas por lazos familiares con los regidores locales, como Nicolás Scorcia para el puesto de Síndico General, y los de Francisco Pascual del Pobil, Nicolás Juan, Francisco Vergara y Carlos Castillo para Diputados del Común, papeletas que se confeccionaban y se distribuían desde la casa del regidor Juan Pascual de Pobil ${ }^{(76)}$. Para el Corregidor, el objetivo era «entrometerse regidores y Alcalde Mayor en el manejo de los propios y arbitrios».

Dos cartas del Conde de Aranda a Juan Gregorio de Muniain, fechadas el 10 y 18 de junio, nos permiten conocer con mayor exactitud la dimensión del problema. En la primera, Aranda considera abusivos los métodos empleados por el Teniente General, a quien critica la utilización de un Ayudante de la plaza, acompañado de un notario, para forzar al escribano municipal a la entrega de una copia de las actas capitulares donde se trató de su sustitución:

«...cuyo exceso es muy notable, porque tira a destruir la libertad que deben tener los regidores para votar y exponer a los Tribunales Superiores lo que juzgan conveniente al servicio del públicon (77).

y en ella también considera una extralimitación del Corregidor en sus funciones haber encarcelado a los regidores Pobil y Rovira. Considera el presidente del Consejo de Castilla que el estado de salud del gobernador lo convertía en persona muy influenciable, y que este tipo de procedimientos extemporáneos «son nacidos de quienes lo gobiernan».

En su segundo escrito a Muniain, el Conde de Aranda era más explícito respecto a la dependencia de terceros del Corregidor. Afirmaba que cuando visitó Alicante en 1765 como máxima autoridad del Reino, observó que el Teniente General José Ladrón no servía para el desempeño de sus funciones por su escasa salud, por lo que había solicitado su retiro, y que se hallaba dominado por su asesor y un grupo de personas, mal vistas por la nobleza local, y que ahora competían con ésta para el control de los nuevos cargos de Síndico y Diputado del Común. Con el ataque de apoplejía y la parálisis de su mano derecha, la estampilla era una peligrosa arma política en manos de la camarilla vinculada al Corregidor que, en opinión de Aranda, «infaliblemente sucederá que la estampilla caerá en sus manos» ${ }^{(78)}$. La posición de Aranda era aconsejar su sustitución y suspender, entre tanto, la concesión de la licencia para el uso de la estampilla. Ninguna de las dos recomendaciones fue atendida, si bien el 21 de junio fue cursada la orden de libertad para los regidores detenidos, orden que cumplimentó de mala gana el Corregidor:

«...no han tenido embarazo (Pobil y Rovira) de aumentar su ofensa con no haberse presentado ni comparecido por mi casa, siendo así que no ha causado poco escándalo verlos correr toda la ciudad» ${ }^{(79)}$. 
El Teniente General Ladrón de Guevara fallecería un año más tarde, siendo Corregidor de Alicante ${ }^{(80)}$.

Un último ejemplo de abuso de poder y menosprecio de las formalidades procedimentales lo aporta el mandato del Teniente General Francisco Pacheco, Corregidor entre 1786 y 1793 . Hombre enérgico, como la mayor parte de quienes ocuparon la Gobernación, tenía iniciativa propia y durante sus años de Corregidor empedró e iluminó algunas calles, creó una Junta de Caridad y logró del obispo de Orihuela la cesión de la denominada Casa de Misericordia para ocupar en ella a pobres en labores textiles ${ }^{\left({ }^{(1)}\right.}$, y persiguió el contrabando con métodos, al parecer, expeditivos ${ }^{(82)}$.

En 1786, el Alcalde Mayor, Luis Gorrón de Contreras, inició contra Pacheco una pesquisa ${ }^{(83)}$ por orden de la Audiencia valenciana. Ésta había recibido un escrito, que después resultaría apócrifo, denunciando irregularidades cometidas por el Corregidor. Los dieciseis cargos contra él resumen el carácter violento, vanidoso y poco dado a formalismos del Teniente General: había golpeado a algunos acusados en juicio; rasgado una pieza de autos y echado el contenido de un tintero sobre una providencia firmada por su asesor de rentas; había utilizado presos en algunas obras públicas, y las había inaugurado con un vítor a su nombre y actuación de la banda de música militar. Pero, sobre todo, se le censuraba inmiscuirse en los procesos, que pretendía fueran vistos rápida y verbalmente en su presencia, sin intervención del Alcalde Mayor ni letrados, expresando públicamente la desconfianza que le merecían abogados y escribanos, quienes en su opinión «enredan los negocios».

En su defensa, el Corregidor justificaba cada una de las acusaciones, aunque admitía su escasa simpatía por las sutilezas del derecho al afirmar que «no puedo dejar de confesar que si en algo no he acertado serán defectos de fórmulas legales, que distan mucho de mi profesión militar» ${ }^{(84)}$.

Campomanes realizó un detallado informe sobre las diligencias actuadas los días 20 y 22 de febrero de 1787 . Reconocía que era criticable su falta de respeto a los trámites judiciales, «gobernando la ciudad del modo que lo podría hacer con la tropa», y consideraba que, pese a ser «negocio de gravedad y de circunstancias trascendentales», no era conveniente proseguir con la capitulación, pudiendo reducirse todo a que el Consejo de Castilla recordara al Teniente General sus obligaciones como Corregidor y magistrado ${ }^{(85)}$.

\section{CONCLUSIONES}

La descripción de algunas de las actuaciones más conflictivas en el trato entre los Corregidores militares y la sociedad civil, ilustra la propensión de los gobernadores a extralimitar sus funciones, y permite aproximarse al conocimiento de formas de oposición política en la vida local valenciana del siglo XVIII de gran interés ${ }^{(86)}$, si bien estos ejemplos que se han señalado muestran únicamente una reducida parcela de la conflictividad política. 
La propia estructura corregimental demandaba el control autoritario de todos los aspectos de la vida local pero, también, la utilización de una red de alianzas y contactos con las élites municipales que sólo se podían desarrollar mostrando una cierta sensibilidad respecto a los intereses locales. Sin embargo, a la vista de los datos que poseemos relativos al perfil profesional de quienes desempeñaron el cargo de Corregidor de Alicante, podemos afirmar que la naturaleza intimidatoria del cargo primó sobre la colaboración con los agentes del poder local, a lo que contribuyó el carácter de una magistratura prácticamente vitalicia y no sometida a responsabilidad civil, lo que hacía difícil no sólo la colaboración fluida con grupos de influencia local, sino la denuncia de abusos y la explicitación de conflictos.

\section{CORREGIDORES-GOBERNADORES DE ALICANTE}

Coronel Patricio MISET (1709-1710)

Brigadier Fernando PINACHO (1710-1715)

Mariscal Juan de CERECEDA (1721-1723)

Mariscal Conde de ROYDEVILLE (1723-1727)

Mariscal Alejandro de la MOTTE (1727-1734)

Brigadier Felipe SOLÍS Y GANTE (1734-1741)

Mariscal Alejandro de la MOTTE (1741-1746)

Mariscal Marqués de ALOS (1746-1760)

Mariscal José LADRÓN DE GUEVARA (1760-1767)

Mariscal Conde de BALLENCOURT (1767-1775)

Teniente General Jorge DUNANT (1775-1783)

Mariscal José Antonio ROMEO (1793-1797)

Teniente General Marqués de ALOS (1797-1798)

Mariscal José de SENTMANAT (1798-1804)

Brigadier José BETEGÓN (1804-1814)

Brigadier José Casimiro de LAVALLE (1814-1815)

Brigadier Fernando de SAINT CROIX (1815-1820)

Brigadier Pedro Fermín IRIBERRI (1823-1832)

Mariscal Isidro de DIEGO (1832-1834) 


\section{NOTAS}

(1) Benjamín GONZÁLEZ ALONSO: El Corregidor Castellano (1348-1808). Madrid 1970.

(2) Para Cataluña, Vid. Joan MERCADER RIBA: Felip Vi Catalunya. Barcelona 1968, pp. 81 y ss.

(3) Mariano PESET: «La creación de la Chancillería de Valencia y su reducción a Audiencia en los años de la Nueva Planta», en Estudios de Historia de Valencia, Valencia 1978, pp. 309-334; Pere MOLAS RIBALTA: «Militares y Togados en la Valencia borbónica», en Actes du I colloque sur le Pays Valencien a l'Epoque Moderne, Pau 1980, pp. 171-186.

(4) Francisco ANDÚJAR CASTILLO: «Aproximación al origen social de los militares en el siglo XVIII (1700-1724)», en Chronica Nova, n. ${ }^{\circ} 10$ (1979), pp. 5-31.

(5) F. FERNÁNDEZ BASTARRECHE: El ejército español en el siglo XIX. Madrid 1978.

(6) Vid. apéndice.

(7) Eduard ESCARTÍN: «El Corregiment de Barcelona: notes per al seu estudi», en Primer Congrés d'Historia Moderna de Catalunya, Barcelona 1984, II, pp. 47-55.

(8) Rogelio SACHÍS LLORENS: Alcoy y la Guerra de Sucesión, 1700-1709. Alicante 1969, pp. 105-107.

(9) Roque CHABAS: Historia de Denia. Alicante 1985 (reed), pp. 366-368.

(10) Jesús PRADELLS NADAL: Del foralismo al centralismo. Alicante 1700-1725. Alicante, 1984, pp. 79-81.

(11) A. H. N. Consejos, Leg. 18.239: Su Majestad al Gobernador del Consejo. 13 de mayo de 1709 .

(12) A. M. A. Privilegios y Provisiones Reales, Arm. 1 Lib. 25, ff. 95-98 v.

(13) El 11 de enero fue designado nuevo Corregidor Luis de Aponte, que era Comandante de la plaza de Cartagena, en sustitución de Patricio Miset, pero este nombramiento no tendría efecto por razones que ignoramos. A. M. A. Privilegios y provisiones reales, Arm. 1, Lib. 25, ff. 268-269.

(14) A. H. N. Consejos, Leg. 17.984: Plan de los Corregimientos que deberán haber en el Reino de Valencia remitida por el Sr. Presidente de aquella Chancillería. Valencia, 20 de noviembre de 1708.

(15) A. H. N. Consejos, Leg. 18.239: Patricio Miset a Juan Milán de Aragón. Alicante, 1 de julio de 1709.

(16) A. H. N. Consejos, Leg. 18.239: Juan Milán de Aragón a Patricio Miset. 13 de julio de 1709.

(17) A. H. N. Consejos, Leg. 17.984: Informe de la Real Audiencia de Valencia firmado por el Gobernador Capitán General Duque de San Pedro sobre los Corregimientos de Valencia y sus territorios. Valencia, 10 de junio de 1721.

(18) A. H. N. Consejos, Leg. 17.985: Mapa de los Corregimientos según deben quedar. Valencia, 30 de septiembre de 1743.

(19) A. H. N. Consejos, Leg. 18.239: Real Decreto designado Gobernador de Alicante al Teniente General Alejandro de la Motte. 2 de septiembre de 1741.

(20) A. G. S. Guerra Moderna, Leg. 1.378: Gerónimo Conde al Marqués de Esquilache. Valencia, 1 de febrero de 1764 . 
(21) A. G. S. Guerra Moderna, Leg. 1.378: El Caballero de la Plaigne al Marqués de Esquilache. Valencia, 4 de febrero de 1740.

(22) A. G. S. Guerra Moderna, Leg. 1.383: Jorge Dunant al Conde de Ricla. Alicante, 8 de julio de 1777.

(23) A. G. S. Guerra Moderna, Leg. 1.383: Jorge Dunant al Conde de Ricla. Alicante, 15 de noviembre de 1777 .

(24) A. G. S. Guerra Moderna, Expediente personal del Teniente General José Chaves.

(25) A. G. S. Guerra Moderna, Expediente personal del Teniente General Alejandro de la Motte.

(26) Fernando SÁNCHEZ MARCOS: «Los oficiales generales de Felipe V», en Cuadernos de Investigación Histórica $n .^{\circ}$ 6 (1982), pp. 241-246.

(27) A. G. S. Guerra Moderna, Leg. 1.855: Súplica del Conde de Roydeville sobre el salario de Mariscal de Campo. Morella, 1721.

(28) A. G. S. Guerra Moderna, Leg. 1.866: Memorial del Mariscal de Campo Alejandro de la Motte. Barcelona, 30 de agosto de 1727.

(29) A. H. N. Consejos, Leg. 18.245: Toma de posesión del Corregidor de Morella. Morella, 10 de septiembre de 1721 .

(30) A. G. S. Guerra Moderna, Leg. 1.279: Duque de Caylus a José Campillo. Valencia, 14 de marzo de 1742.

(31) A. G. S. Guerra Moderna, Leg. 1.598: La Ciudad de Alicante al Marqués de Grimaldo. Alicante, 30 de septiembre de 1715 .

(32) María de los Ángeles PÉREZ SAMPER: «La familia Alós. Una dinastía catalana al servicio del Estado (siglo XVIII)" en Cuadernos de Investigación Histórica, n. ${ }^{\circ} 6$ (1982), pp. 195-239.

(33) A. G. S. Guerra Moderna, Leg. 1.928: Relación de los servicios que tiene hechos a Su Maj. Don Jorge Dunant. Alicante, I de junio de 1777.

(34) María de los Ángeles PÉREZ SAMPER: «La familia Alós...»

(35) A. G. S. Guerra Moderna, Leg. 1.928: Relación de Servicios que tienen hechos a Su Majestad D. Jorge Dunant. Alicante, 1 de junio de 1777; y A. G. S. Guerra Moderna, Leg. 2.662: Hoja de Servicios del Teniente General D. Jorge Dunant. Junio de 1772.

(36) A. G. S. Guerra Moderna, Leg. 1.387: Relación de méritos y servicios del Teniente General D. Antonio Oliver. Alicante. 5 de diciembre de 1784.

(37) A. G. S. Guerra Moderna, Leg. 1.954: Antonio Oliver a Miguel de Muzquiz. San Roque, 14 de enero de 1783.

(38) A. G. S. Guerra Moderna, Leg. 1.311: Marqués de Alós al Marqués de la Ensenada. Napolés, 24 de enero de 1747.

(39) A. G. S. Guerra Moderna, Leg. 6.497: Francisco Javier Pacheco al Conde de Campo Alange. Alicante, 12 de febrero de 1793.

(40) Joan MERCADER RIBA: Felipe V $i$ Catalunya... p. 299.

(41) Eduard ESCARTIN: «El Corregiment de Barcelona...»

(42) El 20 de junio de 1768, el Teniente de Rey dispuso las oportunas órdenes para poner fin a los hurtos que los soldados realizaban en la Huerta. Vid. A. G. S. Guerra Moderna, Leg. 1.380: Órdenes del Teniente de Rey de la plaza de Alicante al Regimiento de Murcia.

(43) A. G. S. Guerra Moderna, Leg. 1.383: José Fernández de Valdivia al Conde de Ricla.

(44) A. G. S. Guerra Moderna, Leg. 1.383: Marqués de Vanmark al Conde de Ricla. Valencia, 23 de noviembre de 1775.

(45) A. G. S. Guerra Moderna, Leg. 1.383: Testimonio de la toma de posesión de D. Jorge Dunant. Alicante, 17 de febrero de 1776.

(46) A. G. S. Guerra Moderna, Leg. 1.960: Jorge Dunant al Conde de Ricla. Tarragona, 5 de septiembre de 1775 . 
(47) A. G. S. Guerra Moderna, Leg. 1.385: Jorge Dunant al Conde de Ricla. Alicante, 22 de abril de 1780 .

(48) A. G. S. Guerra Moderna, Leg. 1.385: Informes contra los excesos del Secretario del Gobernador de Alicante. 1782.

(49) A. G. S. Guerra Moderna, Leg. 170: Consejo de Castilla. 31 de octubre de 1722.

(50) A. M. A. Privilegios y Provisiones Reales, Arm. 1 Lib. 27, f. 312.

(51) A. G. S. Guerra Moderna, Leg. 1.279: Duque de Caylus a José Campillo. Valencia, 14 de marzo de 1742.

(52) A. H. N. Consejos, Leg. 18.239: Real Decreto designando Gobernador interino de Alicante, 20 de julio de 1742; A. G. S. Guerra Moderna, Leg. 1.279: Duque de Caylus a José Campillo. Valencia, 30 de octubre de 1742.

(53) A. H. N. Consejos, Leg. 18.239: Real Decreto designando a D. Antonio Alós Gobernador interino de Alicante. 18 de diciembre de 1746.

(54) A. G. S. Guerra Moderna, Leg. 1.380: Memorial del Teniente de Rey de Alicante José Gutiérrez de Valdivia. 20 de agosto de 1768.

(55) A. G. S. Guerra Moderna, Leg. 1.857: Príncipe de Campoflorido al Marqués de Castelar. Valencia, 19 de abril de 1722.

(56) A. G. S. Guerra Moderna, Leg. 1.857: Juan de Cereceda al Marqués de Castelar. Alicante, 3 de mayo de 1722 .

(57) A. G. S. Guerra Moderna, Leg. 1.857: Juan de Cereceda al Marqués de Grimaldo. Alicante, 3 de mayo de 1722 .

(58) A. G. S. Guerra Moderna, Leg. 1.857: Juan de Cereceda al Marqués de Castelar. Alicante, 8 de junio de 1722 .

(59) A. H. N. Consejos, Leg. 18.239: Real Decreto confiriendo al Gobierno militar y político de Alicante a Don Fernando Pinacho. 15 de marzo de 1710.

(60) A. H. N. Consejos, Leg. 18.239: Cámara de Castilla. 21 de agosto de 1715.

(61) A. M. A. Título y posesión de Antonio Rotlá y Canicia. Arm. 1 Lib. 25, f. 167.

(62) BARÓN DE FINESTRAT: Nobiliario Alicantino. Alicante 1983, pp. 97-101.

(63) El primer Canicia, Juan Bautista, natural de Génova, fue Jurat de Alicante entre 1597 y 1598 y casó con una hija del Marqués del Bosch.

(64) Norbert ELIAS: La Sociedad Cortesana, Méjico 1982, pp. 107 y ss., donde se analiza la conducta de los hombres en la sociedad estamental a través de la etiqueta y el ceremonial.

(65) A. G. S. Guerra Moderna, Leg. 1.375: Memorial de D. ${ }^{a}$ Mariana Vaildo de Llanos. 1758: «Jamás imaginé que pudiera haber hombre, y de las circunstancias y edad del Gobernador que de caso pensado cometiera tan grande desacierto faltando a la veneración y respeto del templo, sin que hagamos mención del que se le debe a una mujer de bien"».

(66) A. G. S. Guerra Moderna, Leg. 1.375: Marqués de Alós a Sebastián de Eslava. Alicante, 8 de febrero de 1758 .

(67) A. G. S. Guerra Moderna, Leg. 1.375: Memorial de D. ${ }^{a}$ Mariana Vaillo de Llanos. 1758.

(68) A. G. S. Guerra Moderna, Leg. 1.375: Marqués de Alós a Sebastián de Eslava. Alicante, 29 de marzo de 1758.

(69) A. G. S. Guerra Moderna, Leg. 1.375: Marqués de Alós a Sebastián de Eslava. Alicante, 19 de abril de 1758.

(70) A. G. S. Guerra Moderna, Leg. 1.375: Dictamen del magistrado Gil de Jaz.

(71) Ibidem.

(72) María de los Ángeles PÉREZ SAMPER: «La familia Alós...» 
(73) A. G. S. Guerra Moderna, Leg. 1.379: Testimonio dado por Nicolás Puigcerver, escribano de número de Alicante. Alicante, 5 de junio de 1766.

(74) A. G. S. Guerra Moderna, Leg. 1.379: La Ciudad de Alicante a D. Manuel de Roda. Alicante, 3 de junio de 1766 .

(75) A. G. S. Guerra Moderna, Leg. 1.379: José Ladrón de Guevara a Manuel de Roda. Alicante, 7 de junio de 1766.

(76) A. G. S. Guerra Moderna, Leg. 1.379: Testimonio de Nicolás Puigcerver, escribano de número de Alicante. 9 de junio de 1766.

(77) A. G. S. Guerra Moderna, Leg. 1.379: Conde de Aranda a Juan Gregorio de Muniain. Madrid, 10 de junio de 1766.

(78) A. G. S. Guerra Moderna, Leg. 1.379: Conde de Aranda a Juan Gregorio de Muniain. Madrid, 18 de junio de 1766.

(79) A. G. S. Guerra Moderna, Leg. 1.379: José Ladrón de Guevara a Juan Gregorio de Muniain. Alicante, 14 de marzo de 1767.

(80) A. G. S. Guerra Moderna, Leg. 1.379: José Gutiérrez de Valdivia a Juan Gregorio de Muniain. Alicante, 14 de marzo de 1767.

(81) Rafael MARTÍNEZ DE SANPEDRO: La Casa de la Misericordia de Alicante. Alicante, 1984. pp. $42-45$.

(82) A. G. S. Guerra Moderna, Leg. 1.387: Memorial de los nobles de Alicante. Alicante, 30 de diciembre de 1786 .

(83) Según el profesor González Alonso la pesquisa es «una investigación limitada y extraordinaria que se centra en la averiguación de hechos presumiblemente delictivos, conocidos merced a la información de determinados particulares y atribuidos a un oficial real al que se suspende en el ejercicio de su cargo mientras la inspección se realiza y se decide su culpabilidad o inocencia. Cfr. Benjamín GONZÁLEZ ALONSO: «Control y responsabilidad de los oficiales reales: notas en torno a una pesquisa del siglo XVIII, en Sobre el Estado y la Administración de la Corona de Castilla en el Antiguo Régimen. Madrid 1981, pp. 203-234.

(84) A. G. S. Guerra Moderna, Leg. 1.022: Memorial de Don Francisco Javier Pacheco. Alicante, 2 de diciembre de 1786 .

(85) A. G. S. Guerra Moderna, Leg. 1.022: El Decano del Consejo en 20 y 22 de febrero de 1787.

(86) El profesor Molas Ribalta estudió desde esta perspectiva la oposición de los gremios de Mataró al Corregidor Betrela y Andrade. Cfr. PERE MOLAS RIBALTA: Societat i poder politic a Mataró, 1718-1808. Barcelona, 1975, pp. 117-131. 